
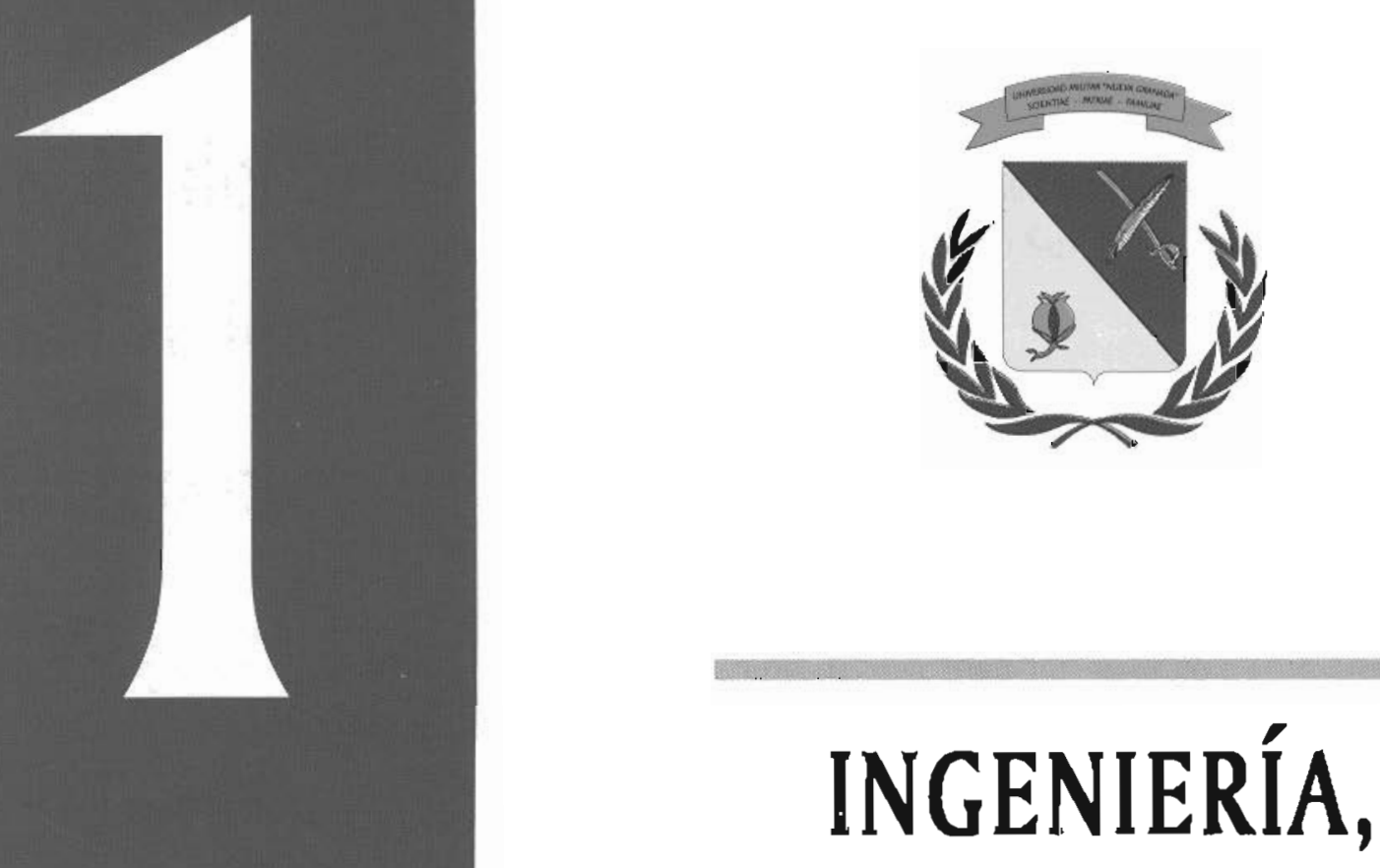
DOCENCIA E INVESTIGACIÓN 


\section{Estado del arte}

del relleno

\section{fluido para}

\section{subbases y}

bases

\section{granulares}

\section{(Primera parte)}

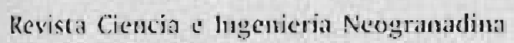
IS5N $012+5170 \mathrm{No}$. 12 - julio de 2002 [Pags. 9-22)

\author{
Luz Elena Santailila Vali:via Ph. D. \\ Rodrico Salamanca Correa ${ }^{2}$
}

$\mathrm{E}$ I articulo recoge una parte del estado del arte del proyecto de investigación "Diseño del moltero fluido para remplazar la subbase y base granular" patrocinado por la Úniversidad Militar Nueva Granada y Concretos Premezclados S.A.. El grupo de investigación esta confomado por la invescigadora principal, la Ingeniera Civil Luz Elena Santaella Valencia Pl..D egresada de la Universidad Francisco de Paula Santander (Cucuta) y docente de medio tiempo de la UMNC. Coimvestigador, Rodrigo Salamanca Correa, Ingeniero $\mathrm{Cl}_{1}$. vil de la Universidad Nacional de Colombia y director de los laborarorios de la Universidad Militar Nueva Granada. Coinvestigador, el Ingeniero Civil Hernán Pimentel, Jefe del Área Técnica de la empresa Concretos Premezclados. Investigadora, Luz Yolanda Morales, Ingeniera Civil, egresada de la Universidad Militar Nueva Granada, quien se ha desempeñado como Jefe del Centro de Investigación de la Facultad de Ingenieria de la UMNG. También se encuentran dentro del grupo ocho estudiantes de noveno y décimo semestre del programa de Ingenieria Civil.

1 Ing. Civil, Coordinadora de la linea de investigación en concreta. Facultad de Ingenieria, Universidad Militar Nueva Granoda.

2 Ing. Civil, Docente de la Facultad de Ingeniería y Director de Jaboratarios de la Facultad de Ingenieria, Universidad Militar Nueva Granada. 


\section{RESUMEN}

El articulo presenta una recopilación de información actualizada sobre el tema de los Rellenos Fluidos, normalmente conocidos en la literatura técnica como 'materiales de baja clensidad y de baja resistencia controladas'. La fimalidad de al informacion es sentar una concepturilización básica sobre el rema, de modo que sirva como punto de partida para un proceso investigarivo que se emprende conjuntamente entre la Universidad Militar "Nueva Granada" y Concretos Premezclados, S.A.. En este trabajo se pretende optimizar tanto el conocimiento como el diseño y el uso aplicado de tal material a las bases y sub-bases de pavimentos, bajo las condiciones de desarrollo recnológico. y de disponibilidad especifica de materiales locales. En consecuencia, se presenta información sobre: generalidades del tema; concepros básicos y las aplicaciones del relleno fluido; los inaceriales componentes: los cliterios de dosificación; las propiedades ranro en estado fresco como endurecido; los conceptos de mezclado, transporte y colocación clel producto; criterios sobre el diseño estruccural del pavinento, y sobre la construcción de los pavimentos, utilizando bases en relleno fluido; el control de calidad, y las veniajas y desventajas asociaclas a la aplicación proptiesta.

Palabras clave: Materiales de baja resistencia controlada (CLSM), relleno nuido sin encoge (RFSE), mortero o relleno fuido, mortero fluido.

\section{SUMMARY}

The article presents an updated compile on 'Fluid Fillers', better known in rechnical reports as 'controlled low density and low strength marerials'. The purpose of the article is to build a basic conceptualisation as a starting point of a research process joincly managed by Universidad Militat "Nueva Granada" and Concretos Premezclados, S.A.. It is the objective to optimise knowledge, design and application of material to base and sub-base in pavements, under local conditions of technical development and avilable row materials. Consequently, information is given as fol- low: introduction: basic concepts and applications of fluid filles's; components; dosage criteria; properties in fresh and hard stacus; mix, transport and placing concepts; criteria on scructural design of pavement, and their coustruction using flud filler; Quality Control; idvantages and disadvantages of proposed ipplication.

Key Words: controlled low strength material (CLSM), backfilling, flowability, flowable fill.

\section{INTRODUCCÓN}

Рara la construcción de un pavimento rígido o flexible se utiliza el miterial granular para la conformación de la base y subbase, mientras que el concreto hidráulico o el asfalto constituyen la capa de rodadura. El material granular de la base o subbase puede presentar grandes deflexiones o incluso hundimientos superiores a $10 \mathrm{~cm}$. con el paso de los vehiculos, debido a factores, tales como: la utilización de maceriales inadecuados según las especificaciones de las normas, una compactación incorrecta por los grandes espesores de capas, equipos inadecuados y deficiente conrol de calidad 228 .

Cuando el material granular no cumple con las especificaciones de la norma, se puede estabilizar con materiales químicos tales como: cemento, cal, cenizas volantes, asfalto, etc. o compactando la arena y la yrava con medios mecánicos, hasta conseguir 100\% del valor solicitado. todo esto retrasa la construcción de la obra

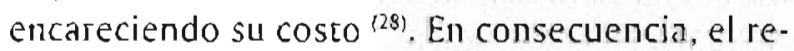
lleno fluido es adecuado para reemplazar la base y subbase granular de un pavimento, aumentando su durabilidad y disminuyendo los costos de construcción y mantenimiento. Sin embargo, las mezclas de relleno fuido no son aptas para resisrir ciclos de hielo-deshielo, fuerzas abrasivas o atacjuc's químicos (19. 16).

Este material recilye otros nombres, cales como: mortero o relleno tuiclo, suelos liquidos o fluidos, relleno fluido que no encoge (RFSE), mor- 
rero o materiales de densidad controlada, mortero o materiales de baja resistencia controlada (CLSM), etc. Los términos reseñados anteriormente describen una familia de mezclas para una variedad de aplicaciones ${ }^{(19)}$. A continuación, se expondrán los conceptos básicos, aplicaciones. materiales utilizados, dosificación, manejo y propiedades de los rellenos fluidos.

\section{Conceptos básicos y aplicaciones del relleno fluido}

Definición. El relleno fluido es un mortero o concreto, mas ligero que los de peso normal, de baja resistencia controlada, que lo hace excilville y una vez endurecido es capaz de soportar cargas mocleradas sin deformarse. De acuerdo con las características propias del relleno, se puede clasificar en dos: relleno de baja resistencia controlada y relleno fiucio de resistencia y densidad controlada.

Relleno fluido de baja resistencia controlada (CLSM). Es un mortero o concreto de baja resisten. cia, de fácil nivelación y aurocompactante, que se puede urilizar en aplicaciones tales como: soleras para tuberías de todo tipo de conducciones o para envolver completamente la tubería de agua potable, aguas residuales, gas, teléfono, energía elćcri$c a$, ecc. Sin embargo, el uso más común del relleno de baja resistencia es como relleno de zanjas, en reemplazo del suelo procedente de la propia excavación o de la utulización de materiales granulares de préstamo, colocándolo directamente del camión a la zanja, se acomoda alrededor de las tuberías brindando un soporte uniforme, sin necesidad de ser compactado ni vibrado, por lo tanto, las dimensiones de la excavación pueden ser múnimas. Después de 5 horas, se puede trabajar encima del relleno fluido, el cual puede alcanzar una resistencia a la compresión de 0.35 a $0.7 \mathrm{MPa}$ similar a la capacidad de soporte del suelo de una zanja. permitiendo ser reexcavado con medios manuales.

Otra utilidad del relleno de baja resistencia es para la subbase y la base de pavimentos, en tráfico ligero de vias urbanas, caso en el cual se vierte directamente entre los bordillos previamente colocados. El espesor de la capa de relleno fluido depende de la resistencia a la compresión del material. Para un rango de resistencias a la compresión entre 3 y $9 \mathrm{MPa}$ los coeficientes estructurales fes una medida de la capacidad relativa de bases y subbases, como un componente estructural de un pavimento flexible) pueden oscilar entre 0.16 y 0.28 , superior al de las subbase y bases granulares y similares a los suelos tratados con cemento (15). Ademas de estos usos, se pueden aplicar para: protección de raludes o cuencos amortiguadores de presas, control de erosión en taludes, caniles y zonas costeras, inyección para relleno de huecos bajo los pavimentos, aceras y losas de transición en aproximaciones a puentes.

Relleno funido de resiscencia y densidad controladas. Si además de la baja resistencia del mortero o concreto se requiere baja densidad, (entre 400 y $1700 \mathrm{~kg} / \mathrm{m}^{3}$ ) esto se consigue añadiendo un adirivo inclusor de aire o agentes espumantes a la mezcla de cemento, árido y agua, obteniendo así los rellenos fluidos de baja resistencia y densidad controlada. Cuando se adiciona el espumante al mortero o concreto, es usual llamarlos concretos o morteros espumados o celulares.

El aire incluido en la mezcla produce una gran cantidad de burbujas con diámetros que van de 20 a 200 micras. Mientrals que en las mezclas con aditivos espumantes, $75 \%$ de las burbujas tienen diámetros entre 0.3 y $1.5 \mathrm{~mm}$., por lo tanto. la estructura y el material endurecido presentan características diferentes, según el aditivo utilizado.

En el relleno de baja resistencia y densidad controlada no es aconsejable utilizar áridos grue. sos, por su tendencia a presentar segregación, aunque es posilsle utilizar áridos ligeros de densidad similar al inaterial endurecido. Estos rellenos se pueden utilizar en todas las aplicaciones anteriores y en otras específicas para reducir e! peso propio de una estructura o en: 
- Azoteas para la formación de pendientes y en cubiertas como capa aislante.

- En estructuras subterráneas de poca profunclidad.

- Tableros de puentes, para rellenar las zanjas donde se alojan las tuberías de servicios.

- Para rellenar los espacios detrás de los muros de contención y de los estribos en puenres.

Como este trabajo está enfocado a la urilización del relleno fluido para bases y subbases, se tendrán en cuenta los materiales, dosificaciones y las propiedades más importantes para el relle no fluido de baja resistencia controlada, que es el más apropiado en este caso.

\section{Materiales para relleno fluido de baja resistencia controlada.}

Los materiales más utilizados en las mezclas de relleno fluido de baja resistencia controlatda son: el cemento porcland con o sin adiciones. agua, aditivo espumante o inclusor de aire, agregados finos, y cuando se requiera mayor resistencia o alta densidad se utilizan agregados gruesos 8. Para seleccionar los materiales se debe considerar la disponibilidad, los costos, la aplicación y las características de fluidez, resistencia, densidad. excavabilidad, contenido de aire, etc. ${ }^{44,19}$.

Cemento. Es el material que suministra cohesion y resistencia a la mezcla, siendo utilizado el cemento con o sin adiciones tipo I y II especiffcado en la norma NTC 121(4. 19.23). También se urilizan los cemencos porrland Tipo IV y V, siempre y cuando los resultados sean aceptables. El contenido de cemento en un inetro cúbico de relleno fluido varia entre 60 y $200 \mathrm{~kg}$, dependiendo de las resistencias reçueridas ${ }^{(31, a)}$.

Agua. Debe ser clara y limpia, libre de sus. tancias perjudiciales para el relleno fuido como aceites, ácidos, álcalis, sales, materia orgánica, etc., es decir, los requerimientos exigidos para el agua que se utiliza en los concretos según la norma NTC 3459 son los mismos que para los morte- ros de relleno fluido ${ }^{123}$. El contenido de agua ell un metro cúbico de mezcla de relieno fluido puede estar encre 140 y 350 . 30. . En las mezclas de CLSM con adiciones de cemizas o con altos contenidos de finos, la cantidad de agua aumenta hasta 620 lirros para alcanzar una buena fluidez. Este rango tan amplio se debe principalmente a las características de los materiales usados en la mezcla de CLSM y el grado de fluidez deseado ${ }^{(4)}$.

Cenizas volantes. Proceden de la combustión del carbón pulverizado en las centrales térmicas y se utilizan las clases $F$ (proceden de la combustión de antracitas y carbones bituminosos) o C (proceden de la combustión de lignitos y carbones subbituminosos) en mayor o menor proporción, con el fin de mejorar la fluidez, incrementar la resistencia a largo plazo y reducir: la densidad. la exudación, la retracción y la permeabilidad de las mezclas ${ }^{(19,32)}$. El uso de las cenizas no es necesario cuando la resistencia del mortero está entre 0.5 y $1.5 \mathrm{MPa}{ }^{(32)}$. La cantidad de ceniza volarte puede estar entre 0 y $120 \mathrm{~kg} / \mathrm{m}^{3}$.

Adítivos químicos. El más utilizado es el inclusor de aire para aumentar la fluidez y reducir tanto la densidad como la resistencia de la mezcla. También se mejoran las propiedades aislintes. como por ejemplo en cubiertas, y se disminuye el contenido de agua en $50 \%{ }^{118)}$. Cuando la mezcla se diseña con suficiente cantidad de funos los conrenidos de are pueden estar entre 15 y 20\% para aumentar la cohesión y evitar la segregación .... También se usan los aditivos espumantes en las mezclas de los rellenos fluidos para producir densidades menores de $1000 \mathrm{~kg} / \mathrm{m}^{3}$, en casos donde se necesita un alto aislamiento 133,34 . Los adicivos acelerantes y reductores de agua se usan en mezclas con bajos comtenidos de finos, para acelerar el fraguado y disminuir el asentamiento ${ }^{18,194}$.

Agregados. Los mús adecuádos son los que cumplen con las normas NTC 174. Los materiales granulares de excavación han mostrado variabilidad en las propiedares fisicas de la mezcla. Mientras que los suelos con finos de arcilla, demandan mayor can- 
tidad de agua, contracción y resistencia variable y la mezcla es pegajosa ${ }^{(19)}$. La cantidad de arena oscila entre los 1400 y $1800 \mathrm{~kg} / \mathrm{m}^{3} 32$. Cuando se quieren obtener resistencias superiores a $3 \mathrm{MPa}$, se utiliza una proporción de agregado grueso con respecto al fino de 1 a 1.4, y siendo el contenido total del agregado del orden de $1400-2100 \mathrm{~kg}^{3} \mathrm{~m}^{3}(32,8)$. Las granulomerias de los agregados para rellemos fluidos que se proponen en el proyecro de norma ${ }^{233}$ deben cumplir los reçuisitos de las Talbas 1 y 2.

Tablo 1. Granulometrias para los agregados utilizados en los rellenos fluidos (23)

\begin{tabular}{|c|c|c|c|c|}
\hline \multirow{2}{*}{$\begin{array}{c}\text { TAMIZ } \\
\text { Pulg (mm.) }\end{array}$} & \multicolumn{2}{|c|}{ ARENA PARA CONCRETO } & \multicolumn{2}{|c|}{ ARENA PARA MORTERO } \\
\hline & Natural & triturado & Natural & Trisurado \\
\hline$=12.5$ & & & . & . \\
\hline$=9.5$ & 100 & 100 & - & - \\
\hline$=4.75$ & $95-100$ & $95-100$ & 100 & 100 \\
\hline$N^{2} 8=2.36$ & $80-100$ & $80-100$ & $95-100$ & $95-100$ \\
\hline $\mathrm{N}^{0} 16=1.18$ & $50-85$ & $50-85$ & $70-100$ & $70-100$ \\
\hline$N^{0} 30=0.589$ & $25-60$ & $25-60$ & $40-75$ & 40.75 \\
\hline$N^{0} 50=0.297$ & $10-30$ & $10-30$ & $10-35$ & $20-40$ \\
\hline$N^{0} 100=0.149$ & $2 \cdot 10$ & $2-10$ & $2-15$ & $10-25$ \\
\hline$N 200=0.074$ & $0-5$ & $0-7$ & $0-5$ & $0-10$ \\
\hline
\end{tabular}

(1) Verificar que los finos proceden de la trituracion.

Tabla 2. Requisitos que deben cumplir los agregados [i.)

\begin{tabular}{|l|r|r|}
\hline REQUISITOS & VALOR MÁXIMO PERMITLO \\
\hline Módulo de finura & & 1.7 \\
\hline Absorción (\%) & & 2.5 \\
\hline Material pasa T200 (\%) & & 7 \\
\hline Materia orgánica & & Escala de color 3 \\
\hline Partículas deleznables (\%) & & 1 \\
\hline Reactividad potencial a los álcalis del cemento. & & Ver NTC 174 y ASTM C289 \\
\hline
\end{tabular}

Cualquier material que se vaya a uritizar es necesario ensayarlo antes de su uso para determinar su aceprabilidad en las mezclas de relleno fluido. Entre los materiales disponibles que se pueden utilizar y son más económicos, están: cenizas volantes con contenidos de carbón hasta de $22 \%$, arena procedente de los procesos de fundición, concreto reciclado, etc. ${ }^{\prime \prime 9}$.

\section{Dosificación de la mezcla}

Actualmente no existe un mécodo especifico de dosificación para e: relleno fluido de baja resistencia controlada (RFBRC); los métodos que se utilizan para morteros y concretos corrientes no son apropiados, por lo ranco, es habirual proceder por tameos hasta conseguir las propiedades deseadas. En algunos trabajos se ha atilizado el método de "Selección de las proporciones para concreto normal en peso $y$ masa, $(\mathrm{ACl} 211)$ " y se ha afirmado que es fiable su uso ${ }^{\text {ii. }}$. El diseño de la mezcla varía según los requerimientos de cada obra, tal como se puede observar en algunos ejemplos de dosificaciones utilizadas en valias obras (18) realizadas en paises como Canadá. Estados Unidos y México, con diferentes ailtelnativas de 
aplicación como se puede observar en algunos de los proyectos que se exponen a continuación;

Caso 1. En Toronto Canadá, el contratista ELES DON realizó un relleno en el paso subterráneo Spadina, debajo de la autopista Halen, que era importante reabrir al tráfico en el menor tiempo posible. La excavación se hizo a más de 15 metros con un volumen total de $7.700 \mathrm{~m}^{3}$ de relleno. Para reducir el tiempo de construcción se utilizó el "relleno fluido que no encoge" (RFSE), con la dosificación que aparece en la Tabla 3 (13).

Caso 2. Se disponia de cuatro horas para rellenar una zanja en una calle de alto tráfico en San Francisco (California). El relleno fluido que no encoge cuya dosificaciön aparece en la Tabla 3 , se colocó a una profundidad de $1.5 \mathrm{~m}$ por encima de la tubería, después de treinta minutos se coloco el geotextil y encima de este, una capa de concreto de $30 \mathrm{~cm}$., que al fraguar lo suficiente, permitió abrir la calle al tráfico a las cuarro horas estipuladas (13).

Caso 3. En las Vegas, se realizó el relleno de una zanja en la cual se colocó una tubería de PVC de $20 \mathrm{~cm}$. de diametro, para proteger unos cables eléctricos que se encontraban dentro de esta. Se utilizó el relleno fluido que no encoge, con un contenido de aire en la mezcla de $30 \%$ y una densidad de $1600 \mathrm{~kg} / \mathrm{m}^{3}$, con el fin de mantener los tubos sumergidos hasta conseguir el fraguado inicial; la dosificación utilizada se puede apreciar en la Tabla 3 \{13\}.
Caso 4. En el sórano del edificio de correos en Boston se quitó un tanque oxidado de combustible. Se exmajeron $615 \mathrm{~m}^{3}$ de tierra contaminada que se reemplazaron con relleno fluido que no encoge (RFSE), que permitió la autonivelación y la apertura del correo ell corto tiempo; la dosificación utilizada se puede apreciar en la Tabla $3^{\text {(13) }}$

La masa total de la mezcla se calcula como la suma de las masas de los componentes. La unidad de medida que se utiliza en las plantas de mezclado para la venta es el metro cúbico de la mezcla de relleno fluido fresco ${ }^{(23)}$.

\section{Propiedades del relleno fluido}

Las propiedades más importantes en las mezclas de relleno fluido de baja resistencia controlada, para bases y subbases de pavimentos son: gran capacidad de soporte estructural, estabilidad de volumen, gran facilidad de colocación y manejo, 1 o se erosiona ell presencia de agua ${ }^{(20)}$.

Las propiedades de una mezcla de relle. no fluido de baja resistencia controlada (RFBRC) es un híbrido encre un suelo y un concreto, por que se fabrica con los materiales usados en el concreto y se coloca de igual manera, pero en servicio sus propiedades se parecen a la de los suelos de calidad controlada. No obstante, los materiales y su dosificación afectan las propje. dades de la mezcla de RFBRC, por lo cual, existe un gran rango de valores para los parámetros que lo caracterizan ${ }^{(19)}$.

Tabla 3. Dosificaciones de RFSE utilizadas en cuatro proyectos "13).

\begin{tabular}{|l|r|r|r|r|}
\hline \multirow{2}{*}{ MATERIALFS } & \multicolumn{3}{c|}{ CANTIDADES DE LOS MATERIALES } \\
\cline { 2 - 5 } & \multicolumn{1}{c|}{ Caso I } & \multicolumn{1}{c|}{ Caso 2 } & \multicolumn{1}{c|}{ Caso 3 } & \multicolumn{1}{c|}{ Caso 4 } \\
\hline Cemento $(\mathrm{Kg})$ & 25 & 120 & 60 & 60 \\
\hline Arena $(\mathrm{Kg})$ & 1365 & 1540 & 1420 & 1400 \\
\hline Agua $(\mathrm{lts})$ & 140 & 145 & 175 & 175 \\
\hline Darafil (ml) & 115 & 115 & 115 & 115 \\
\hline Contenido de aire (\%) & 28 & 20 & 30 & 30 \\
\hline Acelerante (Its) & - & 8 & - & \\
\hline
\end{tabular}




\subsection{Propiedades en estado fresco}

Lintie ellas se discutiran la consistencia o fluidez, el tiempo de fraguado, el contenido de aire, la segregación y contracciôn.

Consistencia o fluidez. Permite que el relleno fluya, se aurocompacte y autonivele, sin requerir la utilización de equipos como sucede con los materiales granulares que se colocan por capas y se compactan mecánicamente. Por lo ranto, la consistencia del relleno fluido puede variar desde plástica hasta fluida, el relleno de consiscencia plástica cuyo rango de asentamiento recomendado está entre 15 y $20 \mathrm{~cm}$. en el cono de Abrams ${ }^{119 !}$, se coloca en las subbases y bases del pavimento que requieren pendientes cercanas a 2\% para el manejo del agua en la superficie del pavimento, $y$ el relleno de consistencia fluida que es autonivelanre, el rango de asentamiento es superior a $20 \mathrm{~cm}$. en el cono de Abrams y se aplica en zanjas ${ }^{(19,20)}$.

Sin embargo, se debe considerar que un relleno muy fluido ejerce una presión hidrostática; para evitarla, es mejo: colocarlo en capas que se dejan endurecer antes de colocar la siguiente. Existen diferentes métodos para determinar la consistencia o fluidez de los RFBRC, que son: el cono de Abrams, el cono de flujo y el ensayo del flujo modificado ${ }^{\{19\}}$.

El ensayo con el cono de Abrams se uriliza de acuerdo con lo especificado en la norma NTC 396 "Ásentamiento de concreto de cemenco portland", cuando la consistencia esperada es menor de $200 \mathrm{~mm}$. y se enmarca dentro de los siguientes intervalos ${ }^{(19.18)}$;

$$
\begin{aligned}
& \text { - Baja fluidez }=15 \mathrm{~cm} \text {. o menos } \\
& \text { - Fluidez normal = entte } 15 \text { y } 20 \mathrm{~cm} \text {. } \\
& \text { - Alta fluidez = superior a } 20 \mathrm{~cm} \text {. }
\end{aligned}
$$

El ensayo con el cono de nujo según la norma ASTM C939 "Flujo de grout para concreto con agregados precolados", se utiliza en mezclas de con- sistencia fluida superior a $200 \mathrm{~mm}$ y y con un tamaño máximo de árido de $6.35 \mathrm{~mm}$. El tiempo recomendado en este método es de $30 \pm 5$ segundos ${ }^{(19,23)}$.

El ensayo de flujo modificado se determina en las mezclas fluidas con predominio de arenas menores a $19 \mathrm{~mm}$. (3/4"), cuando el tamaño de la arena es superior a $19 \mathrm{~mm}$., el ensayo se realiza con el pasalnte del tamiz de $19 \mathrm{~mm}$. y se utiliza un cilindro recto de hierro, plástico u otro material no absorbente, abierto en los extremos, cuyas dimensiones som: $7.62 \mathrm{~cm}$. de diámetro y una altura de $15.24 \mathrm{~cm}$. Se mide el diámetro del material esparcido, que palia los CLSM fluidos, el promedio del diámetro de la rorta típica es de 20 a $30 \mathrm{~cm} .{ }^{18 .}{ }^{19}$.

El ensayo propuesto por el Cuerpo de Ingenieros de los E.U. ell la norma CRD-C611-80 "Método de ensayo para flujo de grout.". se utiliza para mezclas fluiclas con agregados inferiores a $6.35 \mathrm{~mm}$. y el tiempo recomendado en este método es de 12 s (19;,

Tiempo de fraguado. Es el tiempo necesario para que el relleno fuido cambie del estado plástico al endurecido hasta obtener la resistencia para soportar el peso de una persona. El tiempo de fraguado depende del tipo y calidad del cemento, dosificación y fluidez del relleno, temperatura ambiente y de la mezcla, la humedad y el espesor del relleno. En condiciones normales, el paso de personal $y$ máquinas se puede realizar de 3 a 5 horas, y para poder soportar el tráfico normal se requiere de 24 a 36 horas (32): para su determinación se realiza el ensayo de penetración, según la norma NTC 890 cue a su vez nos proporciona la capacidad de soporte del relleno fuido, siendo la más adecuadis una penctración entre 500 y 1500 (19).

Contenidto de aire. Los corntenidos de aire superiores a $20 \%$ disıninuyen significativamence la resistencia a la compresión y facilitan la reexcavación de los rellenos ${ }^{(13)}$.

Scgregación. Las mezclas de alta Ruidez obtenidas con altos contenidos de agua, pueden 
provocar la separación de los componentes; para evitalo es necesario realizar una adecuadal closificación, utilizando materiales finos tales como cenizas volances o limos hasta un $20 \%$ del total de los agregados, para que suministren colsesión. También se pueden emplear conglomerantes con gran contenido de cenizas volantes ${ }^{118.191}$. Se deben evitar los fimos plásricos que incrementan la retracción de la mezcla ir).

Contracción. Se presenta un cambio de volumen por la evaporación del agua, debido a la tomperatura o a lia acción del viento, y a la pérdicla del agua que es absorbida por el rerreno adyacente o es exudada a la superficie, durante el proceso de enchrecimiento de la mezcla. El valar rípico de contracción en mezclas con altos contenidos de agua, está entre 3.1 y 6.35 mm. por cada $30 \mathrm{~cm}$. de profundidad $(19.18)$.

\subsection{Propiedades en estado endurecido}

Entre ellas se verán la resistencia, módulo de rotura, módulo de elasticidad, módulo dinámico, valor relacivo de soporte, contracción, retracción, aislamiento rérmico, excavabilidad, permeabilidad y densidad.

Resiscencia. Para determinar la capacidad que tiene el relleno fluido de distribuir cargas, se realiza el ensayo de resistencia a compresión, la cual debe estar entre 20 y $85 \mathrm{~kg} / \mathrm{cm}^{2}$ para subbase y bases de pavimentos (19l. Según González ${ }^{(16)}$ y Alonso (3). la resistencia a la compresión de una subbase debe estar entre 7 y $14 \mathrm{~kg} / \mathrm{cm} 2 \mathrm{coll}$ un valor relacivo de soporte (VRS) igual o superior a j0" y la resistencia de las bases está entre 15 y 25 $\mathrm{kg} / \mathrm{cm}^{2}$ con un valor relativo de soporte (VRS) igual o superior a $80 \%{ }^{(28)}$. Se permite el uso de un relleno fluido de $85 \mathrm{~kg} / \mathrm{cm}^{2}$ en aquellos sirios donde se presume que no se hará una excavación futura, como puede ser el caso de un relleno estructural

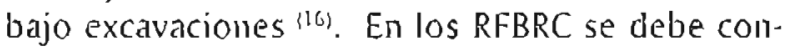
trolar que la resistencia a largo plazo no alcance un valor alto, para que pueda ser reexcavado posteriormente en caso necesario (18).
En los suelos granulares, una resistencia de $50 \mathrm{~kg} / \mathrm{cm}^{2}$ es elevada, ya que la capacidad portante de un suclo granular bien compactado tiene una resistencia a la compresión de 6 a $8 \mathrm{~kg}_{\mathrm{g}} \mathrm{km}^{2}$. mientras que un relleno fluido su resistencia a la compresión máxima especificada es de $85 \mathrm{~kg} / \mathrm{cm}^{2}$. La resistencia del relleno tumbién afecta a los coeficientes escructurales (capacidad relariva de bases y subbases) que oscilan entre 0.16 y 0.28 para una resistencia a la compresión desde $28.84 \mathrm{~kg} / \mathrm{cm}^{2}(19.20\}$.

En consecuencia, la resistencia del relleno fluido puede ser igulal o superior a la del macerial granular compacrado de la subbase y base de un pavimento. El relleno fluido con una resistencia menor o igual a la del material granular que se desea cambiar, se puede colocar en aquellos casos en que se deban mantener los espesores de diseño, y un relleno fluido de mayor resistencia a la clel material granular que se va cambiar, se coloca en el caso que se requiera disminuir los espesores de excavación. Para el cálculo del espesor de una placa de relleno fuido se deben considerar: las cargas de tránsito, CBR, nivel freático, la resistencia, etc. ${ }^{(20)}$.

Una capacidad de soporte de $0.5 \mathrm{Mpa}$ es similar a la de un relleno de cierra con una capacidad de resiscencia de aproximadamence $70 \mathrm{~kg} / \mathrm{cm}^{2}$. En clima caliente, el tiempo para soportar el peso de una persona es de 5 a 8 horas y para soportar las cargas del rráfico es de 24 a 36 horas ${ }^{(13)}$.

El valor de la resistencia a la compresión a la edad estipulada debe ser el resultado del promedio de por lo menos dos cilindros normalizados. La elaboración, curaclo y rotura de los cilindros se debe hacer conforme con la norma NTC673, pero reduciendo la velocidad de carga de la prensa a $0.6 \mathrm{KN} / \mathrm{seg}^{\text {(23). }}$

Módulo de rotura (Mr). En el caso de la resistencia a la flexión del relleno fluido, se ha encontrado que los valores pueden variar entre $10 \mathrm{y}$ $20 \%$ de la resistencia a la compresión. El valor del módulo de rotura para el relleno fluido, se puede estimar con la siguiente ecuación ${ }^{(14)}$ : 
$M r=0.14 *\left(f_{2}^{\prime}\right)$

Donde:

$f^{\prime}{ }^{\prime}=$ Resistencia a la compresión del relleno fluido $\left(\mathrm{kg} / \mathrm{cm}^{2}\right)$

Módulo de clasticidad (Me). Según González los valores del módulo de elasticidad para subbases y bases en relteno fuido se encuentran enrre 16000 y $67000 \mathrm{~kg} / \mathrm{cm}^{2}$, se miden en cilindros de $15 \mathrm{~cm}$. de diámetro y $30 \mathrm{~cm}$. de alrura y se puede aproximar mediante la siguiente expresión, que es válicla para determinar el módulo de elasticidad de rellenos fluidos con densidades entre 1750 y $1950 \mathrm{~kg} / \mathrm{m}^{3}$ (14): $^{4}$

$M e=10235 \cdot f^{\prime \prime}$

\section{Donde:}

$f_{c}=$ Resistencia a la compresion dè relleno fluido $\left(\mathrm{kg} / \mathrm{cm}^{2}\right)$

Mórlulo dinámico (E). Según González (10) los valores del módulo dináınico para subbases y bases en relleno fluido se determinan conforme con la noma ASTM C469, pero debido a la complejidad del equipo pocas veces se realiza, por lo cual son más utilizadas las correlaciones basadas en la resistencia a la compresión y tracción que se calcula con las siguientes ecuaciones:

$E=57000 \cdot \hat{f_{c}^{\prime}}$ para esluerzos de compresión

$E=6670 *(M R)$ para esfuerzos de rracción

\section{Doncle}

$f_{c}=$ Resistencia a la complesión dei relleno fluido (psi)

$M_{k}=$ Múdulo de rotura (psi)

Valor relativo de soporte (VRS) o CBR. Este valor es superior a 100\% a los siete días (según el contenido de cemento) de haber colocado el relleno fluido (16). Pero según Conzález ${ }^{\{6\}}$ el valor relarivo de soporte (VRS) de una subbase debe ser igual o superior a $50 \%$ y la de la base igual o superior a $80 \%$, dependiendo de las condiciones del tránsiro ${ }^{(28)}$. La correlación entre el valor relativo de soporte y la resistencia a la compresión del relleno fluido, se puede calcular mediante la expresión ${ }^{|14|}$

$V R S=2.377 * f_{c}^{\prime}+30.25$

Donde:

$f_{c}=$ Resistcncia a la compresión del relleno thuido $\left(\mathrm{kg} / \mathrm{cm}^{2}\right)$

Contracción. Al contrario que en los reltenos granulares compactados, se ha comprobado que el relleno fluido no presenta asentamiento una vez se ha endurecido (19). El encogimiento (ipico del relleno fluido está entre 0.022 y $0.05 \%$, por lo tanto, no afecta su comportamiento (39.18).

Aislaniento térmico. En este caso se fabri. ca una mezcla con baja densidad y alta porosidad, para lo cual es apropiado utilizal agregiclos livianos y un aditivo espumance, con el fin de utilizarlos para la formación de pendientes en cubierta y en los rellenos que rodean cables de energia elécrica $(19,18)$.

Excavabilidad. Esca posibilidad se ciebe considerar en los proyectos, pensando en futuras reparaciones o renovaciones de servicios (conduc. ciones de agua, gas, electricidad, cables de fibra óptica, erc), ceniendo en cuenta que un relieno fluido con una resistencia a la compresión de 3.5 $\mathrm{kg} / \mathrm{cm}^{2}$ o menos se puede excavar manualmence, y con una resistencias a la compresión de hasta $14 \mathrm{~kg} / \mathrm{cm}^{2}$ es necesario urilizar para su excavación una recroexcavadora, martillos neumáticos o hi. dráulicos. Las mezclas de rellenos fuidos de bajas resistencias con alios contenidos de áridos gruesos son dificiles de excavar a mano $19.18,13$ !

Permcabilidad. La del relleno fluido es parecida a la de los mareriales granulares bien compactados cuyos valores se encuentran enre $10^{-4}$ y $10^{-5} \mathrm{~cm} / \mathrm{sg}$ y los de mayor resistencia y contenidos más alros de finos se obtienen permeabilidades bajas de $10^{-7} \mathrm{~cm} / \mathrm{sg}$. La permeabilidad aumenta a medida que se reduce el contenido de cemento y se aumenta el contenido de agregados que pasan por el tamiz de $80 \mu \mathrm{m}$ (19). 
Densidad. Se determina "in situ" con el densimetro nuclear, cuyos valores se encuencran entre 1800 a $2350 \mathrm{~kg} / \mathrm{m}^{3}$ que es superior a la mayoria de los maceriales granulares bien compactados (18. in 31). Con un contenido de aire de 15 a $30 \%$, se producen densidades húmedas de 1550 a $1950 \mathrm{~kg} / \mathrm{m}^{3}$ cuyo valor en concretos normales es de 2300 a $2500 \mathrm{~kg} / \mathrm{m}^{3}$, siendo myy apco aplicarlo en zanjas ${ }^{13.22)}$. Cuando se utilizan agregados ligeros, cemento y agua las densidades están entre 1441 y $1602 \mathrm{~kg} / \mathrm{m}^{3}(19)$.

\section{Mezclado, transporte y colocación}

Para el procedimiento de mezclado, transporte y colocación de las mezclas de relleno fuido de baja resistencia controlada, por lo general se riene en cuenta el método establecido en la norma $\mathrm{ACl} 304$. Sin embargo, existen otros méto. dos que se han utilizado con resultados aceprables, en los cuales se tiene en cuenta que el relleno sea uniforme. consistente y que cumpla con las caracteristicas esperadas del proyecto ${ }^{(4)}$.

Mezclado. Se puede realizar en centrales de mezclado, plantas de concreto y en camiones mezcladores de concreto. Se debe seguir la misma secuencia y procedimiento de cargue de los maicrijles en todas las bachadas, para asegurar la calidad y uniformidad del relleno fuido, teniendo en cuenta los siguientes pasos (23. 4):

1. Añadir de 70 a $80 \%$ del agua requerida.

2. Añadir $50 \%$ de los agregados finos.

3. Añadir toda la cantidad de cemento y ceniza volante requerida.

4. Añadir la cancidad restante de los agregados finos.

5. Añadir la cancidad restance de agua.

Se debe verificar con antelación el tiempo de mezclado para asegurar la calidad y uniformidad del relleno fluido ${ }^{123)}$. Es fundamencal realizar un buen amasado de la mezcla por un ciempo mínimo de cinco (5) minuros para conseguir la dispersión del adirivo aireante y lograr la fluidez y estabilidad del material (32).

Transporce. Se realiza con camiones mezcladores y/o agitadores. El CLSM requiere que la mezcla se agite comstantemente con el fin de que los componentes sólidos permanezcan en suspensión, durante el transporte y en el tiempo de espera para la descarga. Para distancias cortas $y$ cantidades inferiores a $0.5 \mathrm{~m}^{3}$. el CLSM se ha transportado en equipos sin agitación como camiones de volteo ${ }^{(23,18)}$ a pesar de que los camiones de mezclado suministran un efecto a la mezcla

Colocación. Se puede realizar de forma similar a la de los morteros y concretos corrientes, con los equipos disponibles en las obras, es decir, verrido direcro por la canalera de la mixer, utilización de baldes, cincas o incluso con bombeo a baja presión o con bombas rocativas ${ }^{\text {(32). }}$.

\section{Diseño estructural del pavimento}

Los proyectos de pavimentos se han diseñado con el método de la AASHTO-93, reemplazando la subbase granular por un material cuyo módulo resiliente es de $1.000 .000 \mathrm{lb} / \mathrm{pulg}^{2}$ para conseguir un resultado aproximado a la estructura requerida. Pero el método AASHTO-93 no tiene en cuenta para sus estructuras analizadas un material similar al relleno fluido, por ser un método empírico ${ }^{(16)}$.

En Colombia se ha utilizado el programa DEPAV de la Universidad del Cauca, cuyo procedimiento de diseño se basa en los principios de la mecánica racional de los materiales y la presencia de varias capas ${ }^{(16)}$.

El ayuntamiento de Guadalajara sacó a concurso en 1998 la pavimentación de varias calles, cuya estructura estaba compuesta por una carpeta de $10 \mathrm{~cm}$. de espesor de concreto hidráulico Mr $=50 \mathrm{~kg} / \mathrm{cm}^{2}$, sobre una base de $10 \mathrm{~cm}$. de espesor de relleno fluido de $F^{\prime} \mathrm{C}=25 \mathrm{~kg} / \mathrm{cm}^{2}$. Cemex de México realizó la obra, para la cual determinó el número estructural del pavimento original y el definirivo, reniendo en cuenra los datos que aparecen en la Tabla $4^{(3)}$. 
Tabla 4. Estructura propuesta para los pawimentos compuestos ${ }^{(3)}$.

\begin{tabular}{|c|c|c|c|c|}
\hline $\begin{array}{l}\text { Nimero } \\
\text { capas }\end{array}$ & Coeficiente capa a(i) & $\begin{array}{l}\text { Coeficiente de drenaje } \\
\text { (Cd) }\end{array}$ & Espesor de capa (l) & $\mathrm{a}(\mathrm{i})^{*} \mathrm{Cd}+\mathrm{t}$ \\
\hline \multicolumn{5}{|c|}{ Estructura original propuesta } \\
\hline 1 & 0.50 & 1.00 & 2.95 & $\mathrm{j} .48$ \\
\hline 2 & 0.31 & 1.00 & 1.97 & 0.61 \\
\hline 3 & 0.10 & 0.95 & 5.91 & 0.56 \\
\hline \multicolumn{5}{|c|}{ Número estructura! (SN) $=2.65$} \\
\hline \multicolumn{5}{|c|}{ Estructura final } \\
\hline 1 & 0.50 & 1.00 & 3.94 & 1.97 \\
\hline 2 & 0.22 & 1.00 & 3.94 & 0.87 \\
\hline
\end{tabular}

7. Construcción de pavimentos, colocando una base en relleno fluido

En la construcción del pavimento de siete calles de Guadalajara (México) utilizaron como base el relleno fluido. En una de las calles se pre. sentó agrietamiento plástico del relleno fluido después del fraguado, el cual se corrigió, saturando previamente con aguil la base sobre la que se colocó el réleno fluido y curandio con agua, por lo menos dos veces al día, hasta que se collocara la capa de rodadura (3).

Cuando la capa de rodadura se construye en concreto hidráulico, previamente se debe saturar con agua la base de relleno fluido para prevenir la pérdida de agua del concreto hidráulico y evitar el fisuramiento plástico. También se debe asegurar la adherencia entre estas capas mediante la limpieza de las partículas sueltas y polvo de la superficie del relleno, previo a la colocación de la capa de rodadura. Se recomienda colocar rápidamente la capa de rodadura para proteger el relleno fluido clel medio ambiente y del tränsito de personas y vehículos ${ }^{(3)}$.

Por lo tanto, para evitar la erosión del relleno fluido durante el tiempo que permanezca des. cubierto, se debe tener en cuenta lo siguiente: cerrar los poros del relleno extendido con una llana de aluminio o magnesio hasta obtener una superficie lisa, no dejar circular los vehículos sobre el relleno fluido durante el tiempo que permanezca descubierto y colocar en el menor tiempo posible la capa de rodadura, verificando que los camiones de mezclado no provoquen deformaciones en la superficie del relleno fluido ${ }^{(3)}$.

\section{Control de calidad}

El relleno fluido, por ser un material con gran cantidad de propiedades y usos, requiere un control de calidad de acuerdo con la importancia de la obra, nivel de calidad deseada y de la experiencia que se tenga con el material, para verificar el ajusce de la mezcla con las especificaciones o requisitos.

Para el control de calidad. es necesario realizar los procesos de muestreo. ensayos de conrrol, elaboración y curado de las muestras. El proceso de muestreo lo debe realizar un tomador de muestras, avalado por una entidad certificadil; de igual inanera, el laboracorio que efectue los ensayos debe tener la capacidad de asegurar la calidad y confiabilidad de los resultados de ios ensayos, y dlebe estai certificado por la Superintendencia de Industria y Comercio ${ }^{(23)}$.

Los proyecros de relleno fluido se realizan mediante un diseño de mezclas, empleando las 
materias primas existentes, las cuales para ser evaluadas en estado fresco y endurecido, el productor y el ciience definen el programa de ensayos y escablecen los parimetros, condiciones y rangos de aceptación $(19.23 \%$.

Posteriormente, la verificación en campo se puede realizar visualmente o mediance la realización de ensayos, siendo los más comunes para controlar la calidad del relleno fluido fiesco la consistencia, la remperatura, la masa uniraria, el conrenido de aire, la elaboración y el curado de los cilindros. En obra, los cilindros se deben realizar aleatoriamente cada 40 metros cúbicos de relleno fluido y mínimo una vez al dia. El control de calidad en estado endure. cido se realiza mediante la resistencia, que debe ser el promedio de por lo menos dos cilindros normalizados y representativos de una misma mezcla. El ensayo no se tiene en cuenta cuando la diferencia de los resultados de los cilindros de una misma muestra, ensayados a una misma edad, con los mismos procedimientos. equipos y operarios, supere $10 \%$ de la resistencial media de la muestra (19, 23).

La variación de una bachada se determina para cada propiedad como la diferencia entre el valor más alto y más bajo, obtenidos de las diferentes proporciones de la misma bachada. La comparación se realiza entre clos muestras que representen la prinera y ultima porción de la bachada en ensayo. El relleno fluido se considera uniforme siempre y cuando cumpla la resistencia y con 3 de los 4 ensayos que aparecen en la Tabla 5 (23).
En el rransporte de las muestras de ensayc hacia el laboratorio o sitio de ensayo, se debe evitar al deterioro o alteración de las muestras debido a golpes, vilbraciones, pérdida de hume. dad u orro agenre que altere los resultados de ensayo (23).

\section{Ventajas y desventajas del relleno fluido como base y subbase del pavimento}

Debido a la facilidad y rapidez de colocación del relleno fuido fresco y a las resistencias superiores del marerial endurecido con respecto a los rellenos granulares, se ha podido disminuir las dimensiones de las subbases y bases para pavimentos, disminuyendo la profundidad de excavación de las mismas, permiciendo que su utilización disminuya los costos y tiempos de ejecución de la obra ${ }^{(14)}$.

El Instiruto de Desarrollo Urbano (IDU) de Bogotá está implementando actualmence el uso del relleno fluido en reemplazo de la subbasey base granular cle los pavimentos, debido a las ventajas que este ofrece, tales como:

Gran capacidad de soporte estructural. Es mayor que los rellenos granulares, garantizando un soporte estructural superior a 100\%, a los 7 días de fundido $\langle\mid c\rangle$. También es menos permeable y tiene mayor resistencia a la erosión (31).

Excavable. Propiedad que depende de la resistencia a la compresión de la mezcla de relle-

Tabla 5. Requisitos para la uniformidad del relleno fluida 123 .

\begin{tabular}{|c|c|}
\hline ENSAYO & MÁXIMA DIFERENCIA PERMITIDA \\
\hline Consistencia por el cono de Abranns & $+1-38 \mathrm{~mm}$ \\
\hline Consistencia por cilindro de flujo & $30+1-5 s$ \\
\hline Densidad en estado fresco. $\mathrm{Kg} / \mathrm{m}^{3}$ & 16 \\
\hline Contenido de aire, método de presión, & 2 \\
\hline Resistencia promedio a 7 dias, $\%$ & 3,5 \\
\hline
\end{tabular}


no fluido, que se puede excavar utilizando herramientas manuales como picos o equipos mecánicos (taladros, retroexcavadoras, erc.).

Disponibilidad. Lo fabrica cualquier planta de concreto con materiales focales; el volumen requerido se transporta hasta la obra en camio. nes mezcladores y/o agitadores, eliminando el acopio de material en las vias que permiten la circulación del ráfico y peacones ${ }^{i 3 ?}$.

Puesta en obra. Se puede colocar con canaletas, rampas, bandas transportadoras, bonbas o baldes, no requiere vibración ni compactación: esro se traduce en rapidez, con grandes ahorros en mano de obra y eliminación de los equipo de extendido y compactación $32,1 \%$.

Apertura rápida al tráfico. Por la rapidez en la colocación y capacidad de endurecer, se puede aceptar el tráfico normal, 36 horas después.

Menos controles. Se requieren menos ensavos para comprobar la idoneidad del material, sobre todo cuando este presenta modificaciones en la obra ${ }^{(19.32)}$.

Homogencidad de la mezcla: Se obtiene debilo al proceso de fabricación industrial y a los métodos de ensayos que evalian su calidad e idoneidad (19,32).

La desventaja que presenta el relleno fluido es de tipo económico, ocasionado por el costo de los materiales, pero que se podría equiparar con la subbase y base granular si las estimaciones presupuestales consideraran el mantenimiento que se hace al pavinento mal ejecurado y la re. ducción del espesor de las capas de subbase y base ${ }^{(19,32)}$. Otra desvemiaja que presenta, es la fal ta de disponibilidad en las plantas de concreto del relleno fluido pala utilizarlo en horas nocturnas y dias festivos, debido a los horarios de trabajo de estas plantas.

\section{CONCLUSIONES}

La revisión del tema de los rellenos fluidos, los alcances de su utilización, la experimentación muy variada que sobre ellos se ha efectuado en muy diversos paises, y la base documental y bibliográfica que se presenta, sirven para definir un plan del trabajo de investigación, haciendo énfasis en algunos aspectos, asi:

- La necesidad de realizar ensayos, cuyos resultados prácticos sean aplicables en todo a las condiciones reales de uso en Colombia; prácricos, en cuanto se disenen y evaluen con los materiaies locales, y pricticos también desde el punto de vista comercial, y a costos razonables, para su implementación por parte de la concretera involucrada en la investigación.

- Los resultados esperados después de realizar varios proyectos de investigación relacionados con el relleno fluido, son: 1) definir una metocologia para el diseño de las mezclas de relleno fluido con propiedades similares a una sulubase o base granular de un pavimento rígido o fexible, 2) implementar una serie de elisayos, que por ser frecuentes en otros materiales, tienen normativa y procedimientos muy extendidos, pero sin desconocer las particularidades inherentes a este nuevo material, que aperias se desariolla.

- Se prevé, de acuerdo con la literatura revisada, y con los antecedentes encontraclos, que la urilización del relleno fluido puede ser muy amplia, y técnicanente muy competitiva, para usarse en reemplazo de las bascä y subbases granulares de pavinemos.

\section{BIBLIOGRAFİA}

1. ASOCRETO, "Criterios de control de calidad del concretoy relleno fluido" pp. 21-7. Bogurd.

2. ASOCRETO, "Especificaciones del relleno fluicio". pp. 1-20. Bogora.

3. MI.ONSO OLLINGER, Pedro E.: "Nuevas tecnologias de pavimentación con concreto hidráulico"; CEMEX; pp. 1-19. Bogotä. 1999. 
4. AMERICAN CONCRETE INSTITUTE (ACI) 229R-94, "Controlled low strength materials (CLSM); reported by ACl Committee 229, pp. 55-64, julio, 1994.

5. AMERICAN SOCIETY FOR TESTING AND MATERIAL (ASTM D4832-95): "Standard rest method for preparation and testing of controlled low strength material. CLSM test cylinders"; pp. 319-322: 1998.

6. AMERICAN SOCIETY FOR TESTING AND MATERIAL (ASTM 0597 1-96): "Standard practice for sampling unixed controlled low strength material"; pp. 323-324: 1998.

7. AMERICAN SOCIETY FOR TESTING NND MATFRIAL (ASTM D6023-96): "Standard test method for unit weight, yield. cement content, and air content, gravimetric, of controlled low strength material, CL.SM"; pp. 335-328; 1998.

8. AMERICAN SOCIETY FOR TESTING AND MATERIAL (ASTM D6103-97): "Standard test method for flow consistency of controlled low strength material, CLSM": pp. 332-334; 1998.

9. ADASKA, Wayne S.; "Controlled low strength materials": En: Concrete International-Farmington Hills; N 4 , Vol.19. pp.41-43.195\%

10. NORMA ESPAÑOLA (UNE 83-312-90), "Determinación de la densidad en el hornigon endurecido"; pp. 1-4, 1990.

11. BREWER, William E.; "Results of the Georgetown, Ohio. USA pavement reșearch using controlled low strength maretial, controlled pavement base, CLSM": En: Concrete in the service of mankind: proceedings of the internarional conference; Vol. 5, pp. 253-261; 1996, London. (B1)

12. BREWER. William E.: "Controlled low strength material, CLSM"; En: Concrete in the service of mankind: proceedings of the intermational confercnce: Vol. 5, pp. 655-669; 1996. london. [B]]

13. CODEP-SOCORSA LTDA.; "Fabricación de elcmentos para la industria de la construcción": Representantes del aditivo Darafill. pp. 1-9. Bogorá. (C 1)

14. CEMEX; "Relleno fhuido": En: Revista Constuccion y Tecnologia $N^{\circ}$ 147, Volumen 13. pp. 1-4. México. (C2)

15. FOX, Thomas $A_{i}$ "low strength concrete and controlled low strength material"; En: Transporation research iccord, $\mathrm{N}^{\circ}$ 1234, pp.35-38. Washington, 1989. (F1)

16. GONZÁlez, Carlos Harnán: "Caracterización del relleno flu(io usado como altermativa de rempla\%o de bases $y^{\prime}$ subbases granulares": Concreros Diamanre - Samper; En: XII Simpo. sio Colombiano sobre Ingenieria de pavimentos: pp. 17-1 al 17-14: Julio 4 a! 16, 1999. Medeliin. (6,1)

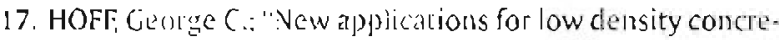
ces": American Concretc listinue (AC!, SP-29): En: Liglitweight concrere, pp.181-220. Detroit, 1971-(H1)

18. JOFRÉ, Carlos; "Rellenos con moreros y hormigones fuludos de baja resistencia controlada"; Revista RUTAS . No7. pp. 5-21, Julio-Agosto, 1998: Madrid (Espana). (J)
19. JARAMILLO PORTO, Diego: "Relleno Fluido. Caracteristicas, propiedades, experiencias": Asociación Colombiana de productores de concreto: ASOCRETO, Jen: Seminario hablemos en concreto sobre pavimentos; pp. 1.21, 1999. Bogotá. (J2)

20. JARAMILlo PORTO, Diego; "Relleno Fluido. Eí nuevo material que reemplaza las bases granulares"; ASOCRETO. pp. 64-68. Bogotá. (13)

21. LARSEN, Ronald L.; "Case studies demonstrating applications of controlied low strength materials": American Concrete Institute (ACI): p.40, 1992, (LI)

22. MATALLANA RODRÍGUEZ, Ricardo: "Rellemo de densidad controlada. Alternativa de cemento pira rellenos de zanjas": En: Boletín ICPC N², julio - diciembre, pp. 9-12. 1998, Medellin. (M1)

23. NTC. Proyecto de norma récnica Colombiana "Especifica" ciones del relleno fluido", pp. 1-8. Bogotá. (N1)

24. NMAl, Charles; MCNEAL, Frances; MARTIN, Dean; "New foaming agent for CLSM applications": En: Concrete InternationalFarmington Hills; $\mathrm{N}^{\circ} 4$, Vol. 19. pp.4447. 1997. (N2)

25. NAIK, Tarun R.; "Low strength concrete and controlled low strength material, CLSM, produced with class fly ash": University of Wisconsin, Wisconsin electric power. Milwaukee, 15p. 1992. (N3)

26. RAMME, Bruce W.; "Progress in CLSM: continuing inmovation"; En: Concrere Internarional-Farmington Hills; $N^{\circ} 5$, Vol.19. pp. 32-33, 1997. (RI)

27. RIGGS, Eugene H.: KECK. Roy H.: "Specificarions and use of controlled low strength material by state transportation agencies": American Society for Testing and Marerials (ASTM STP-1331); En: Design and applications of controlled low strength materiols. flow able fill: pp.296-305, 1998. (R2)

28. RODRŚCUEZ, José: "Áplicación ell vias urbanas. Hormigón ligero para relleno de zanjas": CRASE, S.A.: pp. 179-181. Madrid. (R3)

29. RAMÍREZ GUEJIA, Élmer y SILVA, Javier: "Molteros de relleno con densidad controlada para relleno de tuberias": L:niversidad Militar Nueva Cranada; Facultad de Ingeniéria: pp. 1-17: Bogotá. (R4)

30. RODNíGUEZ JALILI, Arturo; "Relleno fluido. Un material para obras de infraestructura"; En: Construcción y Tecnología $\mathrm{N}^{\circ}$ 143. Volumen 13, pp. 36-40, Abril 20:00, México. (R5)

31. TORRENT PASCUAL. Roberto J; "Rellenos Muidos de Tesistencia controlada"; Instituto Panamericano die Carreteras: IPC. pp. 26-30, 1998, Washington. (T1)

32. VICARIO, Felipe; "Los materiales de baja resistencia contralada (MBRC)": Revista HORMICON N 39 . Volumen 13. p). 39-42, Febrero 1999, Madrid. (VI). 